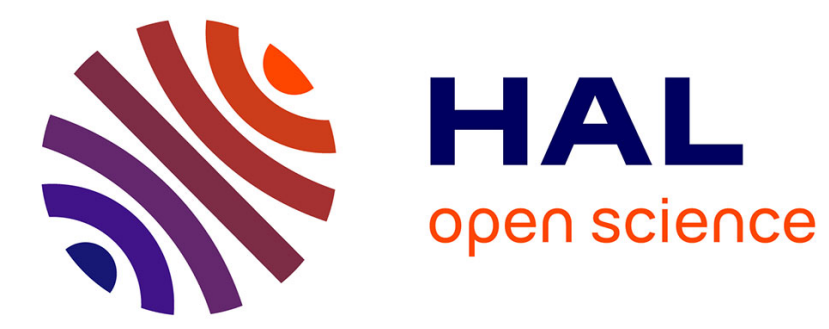

\title{
Rébellion ou soumission à la Cour de cassation ?
}

Anne-Marie Le Pourhiet

\section{To cite this version:}

Anne-Marie Le Pourhiet. Rébellion ou soumission à la Cour de cassation ?. Revue Droit \& Littérature, 2019, 1 (3), pp.45. 10.3917/rdl.003.0045 . hal-02404622

HAL Id: hal-02404622

https://hal-univ-rennes1.archives-ouvertes.fr/hal-02404622

Submitted on 11 Dec 2019

HAL is a multi-disciplinary open access archive for the deposit and dissemination of scientific research documents, whether they are published or not. The documents may come from teaching and research institutions in France or abroad, or from public or private research centers.
L'archive ouverte pluridisciplinaire HAL, est destinée au dépôt et à la diffusion de documents scientifiques de niveau recherche, publiés ou non, émanant des établissements d'enseignement et de recherche français ou étrangers, des laboratoires publics ou privés. 


\section{Rébellion ou soumission à la Cour de cassation ?}

par Anne-Marie Le Pourhiet, professeur de droit public à l'Université Rennes 1

L’ambition affichée du premier président de la Cour de cassation de transformer celle-ci en cour suprême dédiée à la défense des droits et libertés sur le mode de la jurisprudence supranationale consacre moins une rébellion qu'une allégeance conformiste à une contre-révolution politique et juridique. Le remplacement du principe révolutionnaire « La loi est l'expression de la volonté générale " par la devise post-démocratique « Au plaisir du juge » confirme une tendance plutôt réactionnaire apparue en France à la fin de l'ère gaullienne.

Le premier président de la Cour de Cassation, M. Bertrand Louvel, a entrepris depuis sa nomination une auto-réforme de l'institution destinée à en changer complètement l'office afin de mieux appliquer en France le droit international des droits de l'homme véhiculé par les cours européennes et les comités de l'ONU. Cette réforme, défendue à longueur de discours, obéirait, selon son promoteur, à un «darwinisme juridictionnel » qui imposerait à la Cour de cassation de «s'adapter aux besoins de l'homme nouveau, insatiable de libertés » ${ }^{1}$. Se soumettre pour survivre, tel est donc le deal proposé, plus proche de Houellebecq que de Lampedusa.

Cette vaste entreprise a été présentée par un certain nombre de juristes comme une « révolution », tantôt « copernicienne », tantôt simplement «tranquille » ${ }^{2}$. À y regarder de près cependant il est parfaitement clair que loin de s'inscrire dans une démarche de rébellion de juges novateurs décidés à "renverser la table » et à se dresser contre le conformisme, la doctrine ainsi professée apparaît au contraire comme une manifestation d'allégeance, plus réactionnaire que révolutionnaire, à la doxa des cours et des organismes supranationaux. L'idéologie que l'on se propose ainsi d'institutionnaliser s'inscrit dans un mouvement postdémocratique et post-moderne amorcé en France à la fin de la période gaullienne et qui s’est fortement accéléré depuis. Cet alignement juridique est porteur d'une double contrerévolution ${ }^{3}$, à la fois des pouvoirs (I) et des normes elles-mêmes (II).

\section{La contre-révolution des pouvoirs : la post-démocratie}

La réforme annoncée de la Cour de cassation ne constitue qu'une étape supplémentaire dans une longue série d'abdications qui ont fait régresser la France de la souveraineté populaire (A) vers l'aristocratie judiciaire (B).

\section{A. La souveraineté populaire}

\footnotetext{
${ }^{1}$ V. notamment : Bertrand Louvel, «La Cour de cassation face aux défis du XXIe siècle », mars 2015 ; « La Cour de cassation face à l'évolution juridique internationale ", 31 mars 2015 ; "Réflexions à la Cour de cassation », 25 juin 2015, Dalloz Actualité 2015,1326; "Pourquoi je veux une réforme de la Cour de cassation », L'Obs, 11 avril 2018 ; Discours à l'audience solennelle d'installation des nouveaux magistrats, 3 septembre 2018 - www.courdecassation.fr/publications

2 Philippe Jestaz , Jean-Pierre Marguénaud et Christophe Jamin, Révolution tranquille à la Cour de cassation, Recueil Dalloz, 2014, p.2061; Henri de Castries et Nicolas Molfessis (dir.), Sécurité juridique et initiative économique, Mare\&Martin, 2015, p. 146 ; V. aussi Mathieu Delahousse, L'OBS, 11 avril 2018 «Bertrand Louvel a décidé d'imposer sa propre révolution ».
}

${ }^{3}$ François Chénedé, Contre-révolution tranquille à la Cour de cassation, Recueil Dalloz, 2016, p. 796 
La Révolution française, dans le sillage de la modernité, a posé le principe d'un individucitoyen libre, doué de conscience et de raison, qui s’auto-détermine. La réunion de tous les citoyens forme donc une Nation libre, c'est-à-dire souveraine, qui s'auto-détermine également. La conséquence de ce postulat est que les règles applicables dans la société doivent résulter d'un acte de volonté clair des citoyens réunis en corps et c'est donc la loi, votée par eux-mêmes, qui exprimera seule la volonté générale. Ce précepte révolutionnaire s'inscrit à l'article 6 de la Déclaration des droits de l'homme et du citoyen : «La loi est l'expression de la volonté générale. Tous les citoyens ont droit de concourir personnellement, ou par leurs représentants, à sa formation ${ }^{4}$.

La Révolution va donc bannir le droit coutumier, issu des usages traditionnels, ainsi que le droit jurisprudentiel d'origine aristocratique : tout le droit sera désormais contenu dans la loi. Montesquieu lui-même, pourtant convaincu des bienfaits des contre-pouvoirs, avait écrit dans L'esprit des lois : " Dans le gouvernement républicain il est de la nature de la constitution que les juges suivent la lettre de la loi (...). Les juges de la nation ne sont que la bouche qui prononce les paroles de la loi, des êtres inanimés, qui n’en peuvent modérer ni la force ni la rigueur $»^{5}$.

L’idée n'est pas entièrement nouvelle à vrai dire et remonte au droit impérial romain qui apparaît clairement dans les références de Robespierre au code de Justinien et l'on sait que bien avant la Révolution, le monarque souverain avait déjà dû faire face aux velléités de contre-pouvoir des juges, en leur adressant de célèbres remontrances aux remontrances. Louis XIII, dans l'édit de Saint-Germain en Laye de 1641, remettait fermement les parlements, notamment celui de Paris, à leur place, en leur faisant « expresses inhibitions et défenses » de prendre à l'avenir connaissance des affaires qui concernent l'État, l'administration et le gouvernement ${ }^{6}$. Louis XIV, à son tour, dans l'ordonnance civile de 1667 interdit à tous les juges d'interpréter les ordonnances, faisant ainsi application de la maxime romaine ejus est interpretari legem, qui condidit legem («c'est à l'auteur de la loi qu'il appartient de l’interpréter »).

C'est donc immédiatement et dans des termes similaires que la loi révolutionnaire des 16 et 24 août 1790 affirme, dans son article 10 : «Les tribunaux ne pourront ni directement, ni indirectement, prendre part à l'exercice du pouvoir législatif, ni empêcher ou suspendre l'exécution des décrets du corps législatif sanctionnés par le Roi, à peine de forfaiture ». L'interdiction des arrêts de règlement, par lesquels les juges prétendraient statuer de façon générale, est posée à l'article 12 de la même loi et fût même étendue à l'interprétation de la loi qui devait faire l'objet d'un renvoi au législateur. Le rôle du Tribunal de cassation créé par la loi des 27 novembre et $1^{\mathrm{er}}$ décembre 1790 consiste exclusivement à veiller à l'application uniforme de la loi par l'ensemble des juridictions. Il s'agit donc d'une institution révolutionnaire au service de la volonté générale, placée « auprès du corps législatif » et dont les membres sont initialement élus par les citoyens.

La Constitution du 3 septembre 1791 (chapitre V, article 3) reprend les mêmes principes en défendant aux tribunaux de «s'immiscer dans l'exercice du pouvoir législatif ou suspendre l'exécution des lois" et la Constitution de l'an III (article 203) réitère en affirmant: "Les juges ne peuvent s'immiscer dans l'exercice du pouvoir législatif ni faire aucun règlement. Ils ne peuvent arrêter ou suspendre l'exécution d'aucune loi ». C'est sur le

\footnotetext{
${ }^{4}$ Jacques KRYNEN, L'État de justice - France, XIIIe-XXe siècle - tome II - L'emprise contemporaine des juges, Gallimard, NrF, 2012, p. 30 et s.

${ }^{5}$ Livre VI, Chapitre III ; Livre XI, chapitre VI

${ }^{6}$ Édit de Saint-Germain en février 1641 et arrêt du Conseil du Roi du 8 juillet 1661
} 
fondement de ces textes très fermes que la chambre criminelle du Tribunal de cassation s'appuie, le 11 fructidor an V, dans un arrêt Guillaume, pour casser un jugement au motif que le tribunal de police qui l'a rendu «au lieu d'appliquer la loi, seule fonction que la Constitution lui ait déléguée, s’est refusé à ce devoir en se livrant à une critique de la loi qu'il eût du appliquer ${ }^{7}$.

Lorsque le Code pénal de 1810 s’en mêle en décrétant que «seront coupables de forfaiture et punis de la dégradation civique les juges qui se seront immiscés dans l'exercice du pouvoir législatif, soit par des règlements contenant des dispositions législatives, soit en arrêtant ou en suspendant l'exécution d'une ou plusieurs lois, soit en délibérant sur le point de savoir si les lois seront publiées et exécutées ", il témoigne encore, par cette rédaction tendant à couvrir le maximum d'hypothèses, de la volonté de ne laisser aucun angle mort par lequel pourrait se faufiler un contre-pouvoir judiciaire.

Même le juge administratif, apparu plus tard en raison de la prohibition également faite aux tribunaux de s'immiscer dans le contentieux de l'administration, va s'estimer, dans sa sphère de compétence, aussi lié que les magistrats judiciaires par l'interdiction de juger la loi et d'en suspendre l'exécution ${ }^{8}$. Bien que la doctrine publiciste française ait considérablement disserté, sous la IIIe République, sur l'importation en France du contrôle juridictionnel de constitutionnalité des lois, elle n'aura pas convaincu les juges de se lancer dans l'entreprise, alors même d'ailleurs que les lois constitutionnelles de 1875 ne contenaient que des dispositions de procédure et d'organisation, sans aucune mention des droits et des libertés. Dans les fameux arrêts Arrighi et Dame Coudert rendus en 1936, le Conseil d’État rejette encore solennellement un argument tiré de la violation de la Constitution par la loi applicable ${ }^{9}$.

Puis vint la Ve République. Michel Debré dans sa présentation du nouveau texte constitutionnel au Conseil d'État affirme : «L'objet de la réforme constitutionnelle est donc clair. Il est d'abord et avant tout d'essayer de reconstruire un pouvoir sans lequel il n'est ni État, ni démocratie, c'est-à-dire en ce qui nous concerne, ni France, ni République ».

Le Conseil constitutionnel est exclusivement conçu par les auteurs du texte comme un rouage du parlementarisme rationalisé, destiné à encadrer le parlement pour qu'il n'empiète pas sur les prérogatives d'un exécutif qui se veut stable et efficace. Les travaux préparatoires montrent qu'il n'est pas question de lui confier le contrôle du contenu des lois au regard des droits et des libertés mentionnés dans les textes auquel le préambule fait référence. Il doit seulement vérifier que les lois organiques sont conformes aux dispositions constitutionnelles d'encadrement du pouvoir parlementaire, puis contrôler que les règlements des assemblées sont bien conformes aux dispositions précédentes et enfin que les lois votées l'ont bien été en suivant toutes ces règles de compétence et de procédure. Il s'agit d'une pure ingénierie procédurale, exclusive de tout possible jugement de valeur sur le contenu des lois. Le Conseil ne peut d'ailleurs être saisi que par les quatre premiers personnages de l'État : le Président de la République, le Premier ministre, et les deux présidents de chambre.

La loi constitutionnelle du 3 juin 1958 avait bien mentionné parmi les cinq principes qui devraient inspirer la nouvelle constitution, que "L'autorité judiciaire devra être indépendante pour assurer le respect des libertés essentielles définies par le préambule de 1946 et la Déclaration de 1789 ». Mais il s'agit toujours d'assurer ce respect en appliquant la

\footnotetext{
${ }^{7}$ Crim. 11 fructidor an V, Rép. Dalloz, v. Déni de justice

${ }^{8}$ Anne-Marie LE POURHIET, Marbury v. Madison est-il transposable en France ?, in « Marbury v. Madison 1803-2003 », dir. Élisabeth ZOLLER, Dalloz, Thèmes\&Commentaires, 2003, p. 135 et s.

${ }^{9}$ CE, 6 novembre 1936, Sirey, 1937, 3, p.33, concl. Latournerie et note Achille MESTRE ; D, 1938, 3, p.1, note Charles EISENMANN
} 
loi, certainement pas en l'écartant ou en tordant son application. De ce point de vue Michel Debré est encore très clair dans son discours de présentation : "Il n'est ni dans l'esprit du régime parlementaire, ni dans la tradition française, de donner à la justice, c'est-à-dire à chaque justiciable, le droit d'examiner la valeur de la loi ».

Quant à la Convention européenne de sauvegarde des droits de l'homme et des libertés fondamentales adoptée en 1950 dans le cadre du Conseil de l'Europe, de Gaulle en avait refusé sèchement la ratification lors d'un Conseil des ministres où Couve de Murville l'avait fait inscrire, en se disant convaincu par la note de Jean Foyer selon laquelle cette ratification aboutirait à placer la France sous la tutelle des juges européens ${ }^{10}$. De Gaulle dira un jour à ce même Foyer : "Souvenez-vous de ceci : il y a d'abord la France, ensuite l'État, enfin, autant que les intérêts majeurs des deux sont sauvegardés, le droit» ${ }^{11}$ et l'on connaît aussi sa réplique fameuse : "En France, la véritable Cour suprême c'est le peuple ». C'est encore une fois chez de Gaulle que se situe la rébellion, mais ce refus d'adhérer au conformisme démocrate-chrétien ne lui survivra pas.

En mai 1968, la société française remet en cause l'autorité et la hiérarchie sous toutes leurs formes. Le triomphe de l'hédonisme individuel s'accompagne de l'explosion adolescente des «droits » et, par ricochet, de la revanche des juges, dans un cadre européanisé que l’hostilité du gaullisme à la supranationalité avait jusque-là tenu à distance.

\section{B. L'aristocratie judiciaire}

Le président du Sénat, Gaston Monnerville, avait déjà tenté en 1962 de faire censurer la loi constitutionnelle décidant l'élection du président de la République au suffrage direct, mais le Conseil constitutionnel avait sagement refusé de contrôler les lois référendaires exprimant directement la souveraineté nationale. C'est son successeur Alain Poher, peu de temps après la disparition du général de Gaulle, qui prend prétexte d'une loi dite " Marcellin » sur les associations pour offrir au Conseil constitutionnel l'occasion de son premier coup d'État ${ }^{12}$. Le 16 juillet 1971, en effet, le Conseil s'empare du pouvoir de contrôler le contenu des lois qui lui sont déférées par rapport aux droits et libertés auxquels le préambule renvoie. C'est-à-dire qu'il se permet en réalité de changer complètement son office et de tourner désormais son canon contre le pouvoir exécutif puisque, en raison du fait majoritaire, les lois sont désormais l'expression de la volonté du Président exécutée par son gouvernement et votée par sa majorité.

Dans un premier temps, il n’y avait cependant que le Président du Sénat qui était susceptible de contester de temps à autre, devant le Conseil, les choix politiques ratifiés par les trois autres personnalités. La contre-révolution est donc au départ plus qualitative que quantitative. Mais Alain Poher ne se contente pas de poignarder César de l'intérieur, il profite aussi de l'intérim du président Pompidou pour ratifier en 1974 la Convention européenne des droits de l’homme et des libertés fondamentales.

Puis c’est Valéry Giscard d'Estaing qui, en 1974 encore, donne sa bénédiction à la décision de 1971 en étendant le droit de saisine du Conseil constitutionnel à soixante députés ou soixante sénateurs, c'est-à-dire à l'opposition, lui permettant alors de déployer quantitativement le pouvoir conquis trois ans plus tôt.

\footnotetext{
10 Jean FOYER, Sur les chemins du droit avec le Général -Mémoires de ma vie politique, 1944-1988, Fayard, p. 293 et $\mathrm{s}$.

${ }^{11}$ Idem, p. 7

${ }^{12}$ Olivier CAYLA, Le coup d'État de droit ?, Le Débat, n¹00, 1998, p. 108
} 
En 1975, le Conseil sous-entend, dans sa décision sur l'IVG que s'il n'est pas compétent, lui, pour contrôler la conformité des lois au droit européen et international, c'est en revanche aux juges ordinaires, judiciaire et administratif, de s'en charger dans les litiges qui leur sont soumis ${ }^{13}$. La Cour de Cassation ne se fait pas prier et se lance immédiatement, dans l'arrêt Jacques Vabre du 24 mai 1975, dans ce que l’on appelle désormais le «contrôle de conventionnalité » des lois, écartant l'application d'une disposition législative jugée contraire à une règle européenne y compris quand la loi est postérieure à cette règle ${ }^{14}$. La Cour de cassation emprunte d'ailleurs délibérément, dans sa décision, les termes utilisés par la Cour de justice des communautés européennes dans un arrêt de $1964^{15}$. Le Conseil constitutionnel va faire de même en 1988 dans le cadre de son contentieux électoral et le Conseil d'État va suivre en 1989 dans l'arrêt Nicolo ${ }^{16}$.

En juin 1980 la France ratifie aussi les deux pactes de New-York sur les droits civils et politiques et sur les droits économiques, sociaux et culturels. François Mitterrand ratifie encore en 1981 le protocole sur le droit de recours individuel à la Cour européenne des droits de l'homme qui permet à chacun de saisir la Cour après épuisement des voies de recours internes et, en 1983, le protocole sur le droit de plainte individuelle devant le Comité des droits de l'homme de l'ONU. Cette institution n'est pas une juridiction mais se comporte comme telle en rédigeant ses avis comme des arrêts. À partir de ce moment-là tout est en place pour que le gouvernement des juges internationaux prophétisé par Jean Foyer, circule dans l'appareil judiciaire national.

Enfin, le Conseil constitutionnel risquant d'être marginalisé par le contrôle de conventionnalité, Nicolas Sarkozy lui offre en 2008 la question prioritaire de constitutionnalité (QPC) permettant aux justiciables de contester eux-mêmes devant le Conseil une disposition législative dont ils prétendent qu'elle « porte atteinte à un droit ou une liberté que la Constitution garantit». Le terme "prioritaire » signifie que la question de constitutionnalité soulevée dans un procès doit être examinée avant celle de la conformité au droit européen et international. La Cour de cassation, au départ peu favorable à la nouvelle procédure, a d'ailleurs vainement essayé, de faire condamner cette priorité par la Cour de justice de l'Union ${ }^{17}$.

Deux ultimes péripéties se sont encore récemment rajoutées à cet édifice. L’Union européenne s'est dotée d'une Charte des droits fondamentaux annexée au traité de Lisbonne tandis qu'elle doit adhérer elle-même, en vertu du même traité, à la Convention européenne des droits de l'homme, ce qui ne manque pas de déclencher une rivalité entre les deux juges européens de Luxembourg et de Strasbourg. Enfin, le protocole n 16 à la Convention européenne des droits de l'homme permet désormais au juge national de renvoyer à la CEDH les questions d'interprétation de la Convention, ce que la Cour de cassation s'est aussitôt

\footnotetext{
${ }^{13}$ Ccel, 15 janvier 1975, nº74-56 DC

${ }^{14}$ C. Cass. ch.mixte, 24 mai 1975, Société des cafés Jacques Vabre, D. 1975, p.497, concl. Touffait

${ }^{15}$ CJCE, 15 juillet 1964, Costa c/E.N.E.L, aff. 6/64, R.1141 ; Bertrand ANCEL et Marie GORÉ, Marbury v. Madison et l'ordre européen, in «Marbury v. Madison 1803-2003 », dir. Élisabeth ZOLLER, Dalloz, Thèmes\&Commentaires, 2003, p. 151 et s.
}

\footnotetext{
${ }^{16}$ Ccel, 21 octobre 1988, AN, Val d’Oise, $5^{\text {e }}$ circonscription ; CE, Ass. 20 octobre 1989, Nicolo, R. 190, concl. Frydman.

${ }^{17}$ Cass. 16 avril 2010, QPC, nº12003
} 
empressée de faire pour la transcription à l'état-civil de la filiation des enfants issus d'une GPA frauduleuse réalisée à l'étranger ${ }^{18}$.

La boucle est ainsi bouclée : la loi, expression de la volonté générale, est désormais cernée par tous les juges, nationaux comme européens, que leur rivalité va entraîner dans une folle surenchère. En effet, ce droit jurisprudentiel qui l'emporte désormais n'est pas seulement contre-révolutionnaire dans sa source, il l'est aussi dans son contenu. Et c'est bien le but de l’opération : inverser les pouvoirs pour inverser les normes.

\section{La contre-révolution des normes}

Les dispositions constitutionnelles ou conventionnelles qui consacrent des droits et libertés, sont extrêmement vagues et générales. Elles posent toujours, en face des libertés qu'elles consacrent des limites relatives à l'ordre public au sens large. La Déclaration française de 1789 dispose ainsi : "Nul ne doit être inquiété pour ses opinions, même religieuses, pourvu que leur manifestation ne trouble pas l'ordre public établi par la loi ». De la même façon, la Convention européenne des droits de l'homme prévoit-elle, pour chaque liberté qu'elle énonce, de possibles restrictions "prévues par la loi et constituant des mesures nécessaires, dans une société démocratique, à la sécurité publique, à la protection de l'ordre, de la santé ou de la morale publiques, ou à la protection des droits et libertés d'autrui ». Les juges qui prennent en charge l'application de tels textes s'engagent donc dans un contrôle d'opportunité circonstanciel dans lequel une casuistique impressionniste prend le pas sur l'État de droit républicain (A). En outre, ces procédures juridictionnelles sont instrumentalisées par des individus ou groupes qui tendent à faire prévaloir leurs droits subjectifs sur l’intérêt général et la volonté majoritaire (B).

\section{A. La casuistique contre l'État de droit républicain}

Le juge qui s’arroge le droit de vérifier si le législateur national a correctement concilié l'ordre et la liberté, si l'application des dispositions qu'il a édictées pour assurer l'intérêt général ne génère pas une atteinte disproportionnée aux droits et des intérêts privés, ou encore s’il a bien sauvegardé l'équilibre entre deux intérêts privés, substitue immanquablement son appréciation à celle du législateur pour effectuer un contrôle de pure opportunité.

Le juriste autrichien Hans Kelsen qui fût le promoteur du contrôle de constitutionnalité des lois en Europe, avait pourtant prévenu de la nécessité absolue, pour le pouvoir constituant d'éviter la "phraséologie » consistant à écrire des valeurs et des principes vagues tels que liberté, égalité, justice ou équité qui pourraient conduire un tribunal constitutionnel à annuler une loi au motif qu'elle serait simplement injuste ou inopportune. «La puissance du tribunal serait alors telle - écrivait-il - qu'elle devrait être considérée comme simplement insupportable » ${ }^{19}$. C'est précisément ce qu'impliquent les contrôles de constitutionnalité ou de conventionnalité de fond et la vérité est que cette puissance est tout simplement illégitime.

Et que dire des principes de « dignité » ou de «fraternité » créés de toutes pièces par des juges qui peuvent faire dire à ces notions absolument ce qu'ils veulent en se fondant sur le « ressenti » subjectif de plaignants capricieux ? Quoi de plus contraire aux mises en garde de Kelsen que les propos récents du Premier président de la Cour de cassation exposant la nécessité d'adapter l'institution aux «notions coutumières d'équité et de proportionnalité familières au juge anglo-saxon » et imposées par la jurisprudence de la CEDH ?

\footnotetext{
${ }^{18}$ Cass. ass. plén. 5 octobre 2018, nº12-30.138

${ }^{19}$ Hans KELSEN, La garantie juridictionnelle de la Constitution, RDP, 1928, p.p. 240 et 241
} 
La doxa européenne contemporaine consiste à présenter l'existence et la jurisprudence constructive de toutes ces cours comme une garantie de "l'État de droit », alors pourtant que cette casuistique subjective est à l'exact opposé de la notion originelle de Staatsrecht. Les juristes allemands qui ont forgé cette doctrine de droit public l'envisageaient, en effet, comme une organisation hiérarchique des normes et des décisions destinée à éviter l'arbitraire des décideurs et l'insécurité juridique en obligeant les fonctionnaires et les juges à statuer sur la base de lois générales et impersonnelles égales pour tous et connues de tous et non pas en fonction de leur appréciation personnelle. De ce point de vue la doctrine allemande, qui se voulait a-politique, rejoignait cependant parfaitement la conception légaliste républicaine. L’on sait d'ailleurs que les constituants révolutionnaires eux-mêmes avaient agi en députés, et pas seulement en idéologues, lorsqu'ils avaient posé le légicentrisme comme garantie d'égalité et de sécurité. Il s'agissait de satisfaire les nombreuses revendications exprimées dans les Cahiers de doléances en faveur d'une unification législative du droit, seule capable de mettre un terme au désordre normatif textuel et coutumier ainsi qu'à l'arbitraire jurisprudentiel $^{20}$.

Or, précisément, tout ce que propose actuellement le premier président de la Cour de cassation française consiste à changer l'office de son institution pour transformer une cour dont la mission était traditionnellement d'assurer l'égale application des lois, en relais national d'une jurisprudence européenne imprévisible et à géométrie variable qui lui permettrait de recouvrer sa « souveraineté juridictionnelle ». Bertrand Louvel souhaite ainsi instaurer un filtrage des pourvois dont le critère essentiel serait «la défense des droits et des libertés fondamentaux » sous l'égide de la jurisprudence européenne ${ }^{21}$ en revendiquant ouvertement la substitution d'un droit d'inspiration coutumière qui « monte de la société vers le juge » au modèle de "droit écrit qui descend du sommet de l'État centralisé vers le citoyen ». C'est bien le constat d'une inversion contre-révolutionnaire des normes à laquelle il propose de souscrire plutôt que de résister ${ }^{22}$. «Au plaisir du juge » deviendrait ainsi la nouvelle devise de la cassation française, dans un mouvement qu'il faut bien qualifier de réactionnaire.

\section{B. La tyrannie minoritaire}

La CEDH a depuis longtemps montré son adhésion au modèle juridique anglo-saxon et notamment son penchant pour la Cour suprême canadienne. Sa casuistique débridée n’est pas sans rappeler celle des pères jésuites que dénonçait Pascal dans Les provinciales.

La technique canadienne de l'accommodement raisonnable revient en effet à écarter l'application de la norme écrite (loi, règlement administratif, contrat, règlement de copropriété, etc...) chaque fois que son application stricte conduirait à porter une atteinte jugée excessive à un droit subjectif ${ }^{23}$. C'est exactement ce à quoi aboutissent également les

\footnotetext{
${ }^{20}$ Jacques KRYNEN, précité, p. 42

${ }^{21}$ Dîner annuel des juristes franco-britanniques, 31 mars 2015, www.courdecassation.fr/publications

22 Anne-Marie LE POURHIET, La limitation du pouvoir politique : la garantie des droits subjectifs face à la démocratie politique, Dossier «Évolution institutionnelle et normative de la Ve République », Revue française de droit constitutionnel, ${ }^{\circ} 102$, juillet 2015, p. 277 ; L’hégémonie des droits fondamentaux ou l'inversion des fins, in « Les droits fondamentaux, horizon indépassable du droit constitutionnel ? », Revue Politeia, 2016, n³0, p. 317
}

\footnotetext{
${ }^{23}$ Christophe JAMIN, Juger et motiver - Introduction comparative à la question du contrôle de proportionnalité en matière de droits fondamentaux, RTD Civ. 2015, p. 263 et s.
} 
jurisprudences du Conseil d’État et de la Cour de cassation faisant application praeter ou contra legem de la Convention européenne des droits de l'homme ${ }^{24}$.

L'idée qui domine cette jurisprudence compassionnelle est qu'il faudrait écarter l'application de la loi écrite chaque fois qu'elle est jugée " trop dure » pour un individu ou un groupe dont elle contrarie l’identité, la susceptibilité, le désir ou la reconnaissance. Lorsque le parlement national, après débat pluraliste, a posé une règle dans l'intérêt de la société en général, le juge se permet désormais de vérifier qu'elle ne porte pas une atteinte « disproportionnée » à un intérêt individuel ou catégoriel. Le droit au respect de la vie privée et familiale, garanti par l'article 8 de la Convention européenne, sert en particulier de «bonne à tout faire " juridique pour torpiller les dispositions législatives nationales sur la famille et l'immigration et anéantir ainsi la souveraineté étatique. Les valeurs collectives et l'intérêt général défendus par le législateur s’effacent devant la prise en compte du "ressenti » subjectif individuel $^{25}$. L'inspiration multiculturelle anglo-saxonne de cette conception est évidente et l'on a bien compris que le modèle républicain français se trouve dans le collimateur des instances supranationales converties aux droits des minorités ${ }^{26}$. L'État de droit se mue ainsi, selon le bon mot de Guy Carcassonne en "des tas de droits » et le droit public se trouve englouti dans une privatisation tous azimuts où l'adage dura lex sed lex laisse finalement la place à l’injonction « jouir sans entraves ».

Les «méthodes » dégagées par la CEDH et le juge national dans son sillage, consistent à vider les normes de droit public de leur effectivité exactement comme le faisait, au XVIIe siècle, la compagnie de Jésus avec les principes de droit divin ${ }^{27}$. Les pères jésuites auraient ainsi développé, selon les descriptions au vitriol de Pascal, une casuistique laxiste fondée sur toute une série de techniques interprétatives permettant de laisser lettre morte les commandements chrétiens les plus impératifs. Les plus perverses de ces «doctrines » étaient celles de la " grâce actuelle », des « opinions probables », des « circonstances favorables », de la «direction d'intention », de "l'équivoque » et des "restrictions mentales » qui avaient toutes pour objet et pour effet de neutraliser dans des cas concrets l'application de la loi divine en effaçant les péchés les plus énormes. Le parallèle avec les " accommodements » contemporains de la Cour de Strasbourg et de ses relais nationaux est saisissant.

Le sens même de la démocratie est d'ailleurs ouvertement revisité puisque la CEDH n’hésite pas à affirmer, au détour d'une décision, "la démocratie ne se ramène pas à la supériorité constante de l'opinion d'une majorité ; elle commande un équilibre qui assure aux minorités un juste traitement et qui évite tout abus de position dominante ${ }^{28}$. Le mot est lâché : la volonté générale serait un instrument de "domination" qu'une aristocratie judiciaire devrait donc écarter chaque fois qu'une minorité s'estime injustement opprimée par l'intérêt général ${ }^{29}$. Il s'agit bien de satisfaire ce que Marcel Gauchet nomme «les droits de l'individu en société contre la société ", c’est-à-dire le contraire de la république ${ }^{30}$.

\footnotetext{
${ }^{24}$ Voir notamment :

- Cass. civ. 4 décembre 2013, n¹2-26-066, Bull.civ. n²34 ; D. 2014, 179, note François CHENEDÉ

- CE, Ass. 31 mai 2016, Mme Gonzalez-Gomez, n³96848, RFDA, 2016, n4, p. 754, note Pierre DELVOLVÉ

${ }^{25}$ Marcel GAUCHET, Que faire des droits de l'homme ?, Revue des deux mondes, février-mars 2018, pp. 8 et s.

${ }^{26}$ Voir notamment : ONU - Assemblée Générale, Conseil des droits de l'homme, Rapport McDougall, A/HRC/7/23/Add. 2 - 3 mars 2008

${ }^{27}$ Blaise PASCAL, Les Provinciales, Édition de Michel Le Guern, Gallimard, Folio Classique, 1987

${ }^{28}$ CEDH, Young, James et Webster, 13 août 1981, série A, n4

${ }^{29}$ Andrée LAJOIE, Quand les minorités font la loi, PUF, Les voies du droit, 2002

${ }^{30}$ précité p. 12
} 
Lors des débats accompagnant l'adoption de la question prioritaire de constitutionnalité (QPC), s'est également répandue en France une rhétorique langagière vantant les mérites d'une procédure permettant enfin aux citoyens de «s'approprier la Constitution ». Nul n'a songé à s'arrêter sur cette expression ni sur ce qu'elle dit exactement de la privatisation de la norme commune, de la captation féodale de la Res Publica et de la transformation du citoyen en ayant-droit. Le doyen Carbonnier avait pourtant alerté dès 1996 sur les dangers de la "pulvérisation du droit objectif en droits subjectifs » ${ }^{31}$ à laquelle travaillent aujourd'hui ardemment les juges français.

Dans ces conditions la loi votée par les assemblées législatives élues est désormais vouée à l'instabilité et l'insécurité puisqu'en permanence susceptible de voir son existence ou son application remise en cause. Les citoyens qui votent pour des candidats présentant un programme législatif sont assurés que celui-ci se retrouvera en réalité sous la tutelle de juges qui décideront finalement discrétionnairement de son effectivité. L’isoloir est marginalisé puisque tout se passe in fine dans le prétoire et la majorité des citoyens voit sa volonté anéantie par les intérêts de quelques ayant-droits bénéficiant de la complaisance des juges. L'on comprend bien le sentiment de dépossession qui s'empare des peuples dans un système normatif où le déficit démocratique est délibérément organisé et revendiqué à l'aide d'une véritable annulation des résultats des élections ou des référendums par des oligarchies de toutes sortes.

C’est assurément contre l'attitude anti-républicaine et réactionnaire des juges que devrait s'organiser la véritable rébellion des citoyens. Certains juristes ont commencé à s'insurger contre la nouvelle tentative de coup d'État juridictionnel ${ }^{32}$, il convient assurément de poursuivre dans cette voie en défendant les acquis juridiques de la grande Révolution. Il sera peut-être nécessaire d'en passer, pour ce faire, par la révision constitutionnelle et/ou la dénonciation conventionnelle.

\footnotetext{
31 Jean CARBONNIER, Droit et passion du droit sous la Ve République, Flammarion, 1996

32 Bernard HAFTEL, Lucie MAYER et autres, Non à la transformation de la Cour de cassation en « Cour suprême », Les Échos, 19 juillet 2018 ; Voir aussi : Dossier, Regards sur la réforme de la Cour de cassation, Revue de droit d’Assas, n¹6, mai 2018, pp. 46 et s.
} 